\title{
Ciencia, educación y sociedad: temas de actualidad para la realidad mexicana
}

\author{
Víctor Jesús Rendón Cazales
}

Como parte de la realidad actual de México y de muchos países del mundo, el interés en el desarrollo científico, la transformación educativa y los diferentes procesos sociales, se ha puesto de manifiesto dada la necesidad de implementar cambios en las condiciones sociales y de construir futuros distintos. El desarrollo tecnológico, su aplicación a procesos sociales, el cuidado y mejora del medio ambiente, son temas recurrentes que los distintos actores universitarios ponen sobre la mesa, realizando diversos tipos de acciones desde sus trincheras, una de ellas, la difusión y puesta al escrutinio de sus reflexiones y hallazgos. Es por eso que el presente número de la Revista Digital Universitaria (RDU) presenta una serie de textos, con diferentes problemáticas, que muestran un mosaico de aportaciones.

La innovación tecnológica y las transformaciones deseables, derivadas de los proyectos de investigación e intervención, resultan de gran relevancia desde el quehacer universitario, dado el papel que las instituciones de educación superior (IES) tienen en la generación de nuevo conocimiento y la mejora de las condiciones sociales. Aunado a esto, la vinculación con el trabajo que desarrollan otras universidades en diferentes partes del mundo permite que nuestro país se coloque en las discusiones internacionales sobre las situaciones que aquejan a las sociedades, pero, lo más importante, posibilita que las aportaciones generadas contribuyan a la solución de problemas. Este interés se ve reflejado en cada uno de los artículos que para este número la RDu pone a disposición de los lectores, ya que se abordan temas desde las ciencias "duras", hasta la educación y sus intersecciones, con miras a proponer mejoras en las realidades sociales.

Las cuatro secciones de este número —Varietas, Continuum educativo, Universidades y Caleidoscopio - nos ofrecen la oportunidad de aprender sobre problemas educativos relacionados con tecnologías educativas, ambientes virtuales, métodos de enseñanza o trabajo comunitario; así como sobre el desarrollo tecnológico y su relación con la prevención de sismos, métodos alternativos de producción, ecológicamente sustentables, el comportamiento fisicoquímico de los elementos o la estructura genética. Todo esto con el propósito de brindar conocimiento a la sociedad sobre fenómenos de interés y así tener la posibilidad de tomar

Dol: http://doi.org/10.22201/cuaieed.16076079e.2020.21.6.0 
decisiones fundamentadas en la evidencia científica. A continuación, se describe brevemente el contenido de cada una de las secciones que conforman la emisión correspondiente a noviembre-diciembre de la RDU.

En la sección Varietas dos trabajos abordan problemas educativos que buscan mejorar procesos y prácticas en las aulas universitarias. El primero, titulado "Tecnologías para la comprensión lectora: estado actual y nuevos desarrollos", aborda el impacto de la tecnología en el desempeño en habilidades de comprensión lectora, en particular, los sistemas de tutoría inteligente. Este tipo de desarrollos tecnológicos pretende apoyar las prácticas educativas al retroalimentar y ofrecer comentarios individuales a las necesidades de cada estudiante, a partir de los algoritmos de la inteligencia artificial. Por su parte, el artículo "La historia es de quien la trabaja. Documentos indígenas: una ventana al pasado" aborda una manera alternativa y atractiva de la enseñanza de la Historia, a partir del estudio de los títulos primordiales de los pueblos, es decir, de los primeros textos que se escribieron en una localidad y que forman parte de su legado, memoria e identidad histórica que los diferencia de los demás. El trabajo "Experiencias de docencia, extensión e investigación para la promoción de lactancia y donación de leche materna" ofrece una visión con un impacto social mucho más cercano a los beneficiarios, al abordar uno de los temas muy poco estudiados: la donación de la leche materna en Argentina. En esta publicación se busca mostrar cómo los vínculos entre la investigación, la docencia y la comunidad pueden funcionar para mejorar las condiciones de personas con mayor necesidad de atención.

Los otros textos que componen esta sección abordan temas derivados de la Biología, Geología, Genética y Química. Así, dos trabajos se enfocan en la generación de nuevos conocimientos disciplinares como el artículo "La tabla periódica y sus patrones para la predicción del comportamiento fisicoquímico" y el trabajo titulado "Epigenética: candados y llaves durante la lectura del adn". La primera de estas contribuciones ofrece elementos para la consideración de la tabla periódica y sus patrones de predicción del comportamiento fisicoquímico de los elementos; mientras que el segundo brinda conocimiento alrededor de la epigenética como factor en la regulación de la expresión genética de las células. Por otra parte, los artículos "Adecuación de la Ley de Gutenberg. Richter al contexto mexicano" y "Piscicultura marina tropical con peces diádromos: estrategia frente al cambio climático en México" ofrecen resultados dirigidos a solucionar problemas naturales y ambientales que aquejan a las sociedades. Así, el primero se enfoca en el estudio de los sismos y la prevención de los desastres derivados de ellos, mientras que el segundo aborda una alternativa en la producción pesquera ante las consecuencias del cambio climático provocado por la mano humana. Por último en esta sección, en el texto "Una competencia de pesos pesados por el dominio del mundo", se compara la biomasa de los seres humanos contra la de las plantas, los microorganismos y las vacas, para, a través de 3 rounds de lucha, darse cuenta de que los seres humanos no somos, en definitiva, la especie dominante del planeta. 
En la sección de Continumm educativo, encontraremos diversos artículos enfocados en resaltar usos de herramientas digitales en contextos de educación. Éstos cobran especial relevancia dadas las circunstancias actuales en la pandemia por el coronavirus, SARS-CoV-2, la cual ha transformado diversos ámbitos de la vida social, entre ellos la educación. Los dos primeros textos titulados "Uso tecnopedagógico de dispositivos móviles en la formación de investigadores" y "Realidad aumentada en la enseñanza" ponen a discusión el uso de dispositivos tecnológicos que en un principio parecieran en polos extremos en cuanto a su complejidad y su disponibilidad: el teléfono celular y una aplicación basada en la realidad aumentada implementada en el Taller de Cómputo del Colegio de Ciencias y Humanidades. Ambas contribuciones buscan descubrir los efectos pedagógicos de estas herramientas en cuanto al acceso a la información, la colaboración, la manipulación de objetos digitales, entre otras cuestiones. Finalmente, el trabajo "Diseño de un blog para la divulgación de contenidos educativos relacionados con nanociencia y nanotecnología" aborda la utilidad de un blog como espacio digital para compartir materiales educativos de diferentes experiencias, relacionadas con la enseñanza de nanociencia y nanotecnología en Colombia. Este esfuerzo es una manera de documentar cómo en este espacio se puede colaborar y diseminar los conocimientos entre diversos investigadores.

La sección Universidades retoma tres trabajos enfocados en diferentes dimensiones educativas. Por un lado, "Los FabLab y el Design Thinking: nuevas estrategias para el aprendizaje creativo" especifica una experiencia en el aula empleando una metodología de intervención pedagógica (Design Thinking), como estrategia para favorecer el pensamiento creativo. Por su parte, "Panorama del servicio de referencia digital en bibliotecas de la unam" nos ofrece un análisis sobre las condiciones y características de las bibliotecas digitales de la Universidad, lo cual se encamina a la mejora de los servicios educativos que se ofrecen en esta casa de estudios. Por último en esta sección, en "Políticas educativas e incorporación de las tic en la educación superior mexicana", encontramos un análisis documental crítico sobre la introducción de las Tecnologías de Información y Comunicación (TIC) en el sistema educativo mexicano, plasmado en diversos textos de políticas públicas, desde donde se plantea enfatizar el acceso a las prácticas sociales y los procesos educativos por encima de reducir las acciones a la disponibilidad de los dispositivos tecnológicos.

Como complemento a las secciones anteriores, en Caleidoscopio, en el artículo "Creencias ambientales mexicanas. La importancia de construir sociedades resilientes", se reseña el libro La dimensión ambiental en los albores del sig/o xxl, donde el autor nos muestra los claroscuros de las opiniones de la población en relación con el tema del medio ambiente en México. Esta reseña nos abre un panorama del libro original y nos invita a reflexionar sobre las relaciones que tenemos con el medio natural, los demás organismos vivos y las creencias que ante los temas ambientales. 
Como se puede apreciar, las contribuciones de los autores y autoras que participaron en la conformación de este número de la RDu son una referencia para aquellos interesados en conocer temas de actualidad, que tarde o temprano tenemos que poner a discusión. El interés por la mejora de los procesos educativos no sólo implica la introducción de tecnologías, sino la de sus usos, así como comprender sus posibilidades para construir nuevas realidades sociales. Uno de los propósitos que se busca lograr en los procesos educativos tiene que ver con la mejor convivencia entre los actores sociales, pero también con el medio ambiente, de tal forma, que realicemos prácticas sustentables que nos permitan una relación más armoniosa con éste. Para ello, es importante retomar el desarrollo teórico y metodológico que se lleva a cabo en diversas disciplinas, así como acercarnos a los diferentes medios de difusión con los que contamos, entre ellos el trabajo bimestral que realiza la Revista Digital Universitaria.

\section{Cómo CITAR ESTE ARTículo}

* Rendón Cazales, Víctor Jesús. (2020, noviembre-diciembre). Ciencia, educación y sociedad: temas de actualidad para la realidad mexicana. Revista Digital Universitaria (RDU), 21(6). Dol: http://doi.org/10.22201/cuaieed.16076079e.2020.21.6.0

\section{DATOS DE AUTOR}

\section{Víctor Jesús Rendón Cazales}

victor rendon@cuaieed.unam.mx

Doctor en Ciencias con especialidad en Investigaciones Educativas por el Departamento de Investigaciones Educativas del Cinvestav-IPN. Especialista en temas relacionados con prácticas sociales de lectura y escritura, cultura digital, desarrollo profesional docente y vinculación entre educación formal y no formal. Ha participado en diferentes proyectos de investigación y tiene publicaciones relacionadas con la apropiación de tecnologías digitales en ámbitos educativos. 